\title{
Normal mode calculations of trigonal selenium
}

\author{
Hansen, Flemming Yssing; McMurry, H. L.
}

Published in:

Journal of Chemical Physics

Link to article, DOI:

10.1063/1.438973

Publication date:

1980

Document Version

Publisher's PDF, also known as Version of record

Link back to DTU Orbit

Citation (APA):

Hansen, F. Y., \& McMurry, H. L. (1980). Normal mode calculations of trigonal selenium. Journal of Chemical Physics, 72(10), 5550-5564. https://doi.org/10.1063/1.438973

\section{General rights}

Copyright and moral rights for the publications made accessible in the public portal are retained by the authors and/or other copyright owners and it is a condition of accessing publications that users recognise and abide by the legal requirements associated with these rights.

- Users may download and print one copy of any publication from the public portal for the purpose of private study or research.

- You may not further distribute the material or use it for any profit-making activity or commercial gain

- You may freely distribute the URL identifying the publication in the public portal

If you believe that this document breaches copyright please contact us providing details, and we will remove access to the work immediately and investigate your claim 


\title{
Normal mode calculations of trigonal selenium
}

\author{
Flemming $Y$. Hansen \\ Fysisk-Kemisk Institut, The Technical University of Denmark, DK 2800 Lyngby, Denmark
}

H. L. McMurry ${ }^{a)}$

Research Reactor Facility, University of Missouri, Columbia, Missouri 65211

(Received 11 May 1978; accepted 5 February 1890)

\begin{abstract}
The phonon dispersion relations for trigonal selenium have been calculated on the basis of a short range potential field model. Electrostatic long range forces have not been included. The force field is defined in terms of symmetrized coordinates which reflect partly the symmetry of the space group. With such coordinates a potential energy, calculated with only a diagonal force matrix, is equivalent to one calculated with both off diagonal and diagonal elements when conventional coordinates are used. Another advantage is that often some force constants may be determined directly from frequencies at points of high symmetry. The intrachain force field is projected from a valence type field including a bond stretch, angle bend, and dihedral torsion. With these coordinates we obtain the strong dispersion of the upper optic modes as observed by neutron scattering, where other models have failed and give flat bands. The interchain force field is projected from relative rotations and translations of groups of atoms in adjacent chains. This type of coordinate is very well adopted to describing interactions between groups of nonbonded atoms as found in molecular crystals, and they also seem to apply very well for this crystal. In this way we have eliminated the ambiguity in the choice of valence coordinates, which has been a problem in previous models which used valence type interactions. Calculated sound velocities and elastic moduli are also given.
\end{abstract}

\section{INTRODUCTION}

In this paper we analyze the dynamics of trigonal selenium based on the recent rather complete neutron scattering data in various symmetry directions. ${ }^{1,2}$ These data provide a good basis for a thorough test of force models.

The first attempt to set up a force model for trigonal selenium was done by Hulin. ${ }^{3}$ This work was later extended by Geick et al. , ${ }^{4}$ who proposed a Born-von Kármán force constant model with general forces in the chain and a central force between close neighbors on adjacent chains. At that time only two elastic constants and optic data at the zone center $\Gamma$ were available and this severely limited tests of the models. However, even without data these models were seen to have a serious defect at the zone boundary $\left[\frac{1}{3}, \frac{1}{3}, 0\right]$, where one of the $K_{2}$ frequencies collapsed to zero. If symmetry coordinates which block diagonalize the secular equation are used it becomes clear that the restriction to central forces between nearest neighbors on adjacent chains will always produce this collapse. This shows at once that a more elaborate force field is a physical requirement in crystals of the trigonal selenium structure. Geick et al. ${ }^{4}$ suggested introducing a central force between fourth neighbors. Wendel et al. tried this ${ }^{5}$ and, while the collapse could be avoided, the calculated dispersion relations differed considerably from the experimental results. In the process of improving the fit to the experimental results, they found that the formal Born-von Kármán force constants were extremely dif ficult to handle, since the parameters seemed to be correlated in a nontransparent way. Nakayama and Odajima ${ }^{6}$ used a simple valence force model for all in-

\footnotetext{
a) Present address: Department of Physics, Idaho State University, Pocatello, ID 83209.
}

teractions. The interchain interactions used bond stretches between nearest neighbors and bending of the angles between such bonds. The interspiral interactions used bond stretches between closest neighbors in adjacent cells. They obtained good results for the $\Gamma$ frequencies apart from the $\Gamma_{2}$ frequency. Their calculation necessarily had the collapse of the $K_{2}$ frequency. In a later paper ${ }^{7}$ this was eliminated by the introduction of one more stretch coordinate between nextnearest atoms in adjacent cells (equivalent to interactions between fourth nearest atoms). The upper optical modes were flat.

Martin et al. ${ }^{8}$ extended the valence force picture of Nakayama et $a l$. by including angle bend coordinates in the interchain force field. This eliminates the collapse of the $K_{2}$ frequency in a more physically reasonable way. The difficulty in the method used by Martin et al. lies in the fact that there are several angles of different magnitude to consider, and they neglect some angles and use the same force constants for angles of different sizes. They obtain good results for the acoustic and low optical modes, whereas the upper optical modes are flat in contradiction to the experimental results. Wendel et a $l .^{5}$ treated the problem in another way. They worked with a combined valence force field and a Keating potential ${ }^{9}$ field, which gave them the capacity to recognize the different angles without using the same force constants for them and at the same time keeping the number of force constants at a manageable level. They obtained good results for the acoustical and low optical modes, but the upper optic modes were flat. To improve this deficiency of their model, they investigated various shell models for the Coulombic forces, which are present in selenium. They noted some improvement but not to the degree demanded by the experimental results.

The potential field used here differs in two essential 


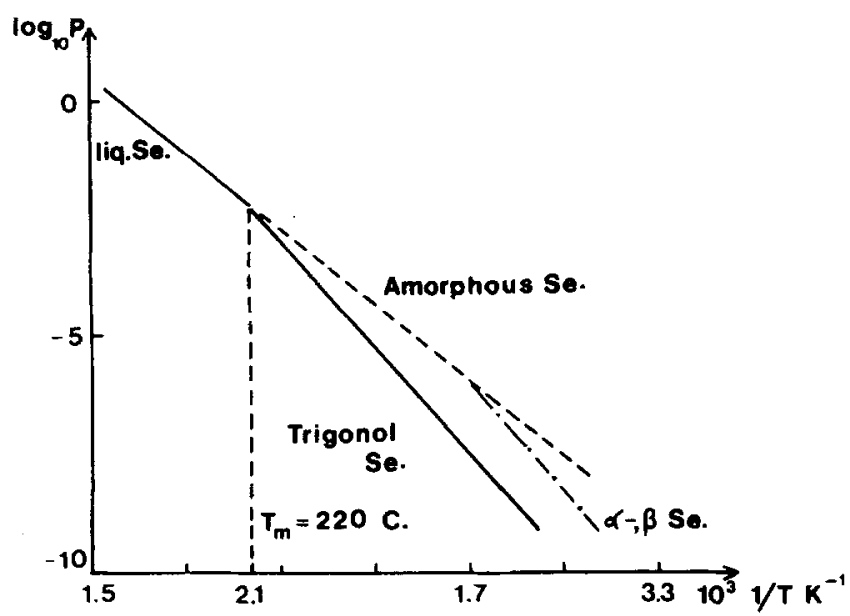

FIG. 1. Phase diagram of selenium.

ways from the fields just discussed. We follow the procedures developed in the accompanying paper ${ }^{10}$ (hereafter referred to as I) and use symmetrized valence coordinates for the intrachain interactions (including dihedral torsions) and symmetrized relative motion coordinates for the interchain interactions. The symmetrized intrachain dihedral torsions prove to be necessary to give the dispersion of the upper optic modes in directions along the screw axis and have not been included before.

We prefer the relative motion coordinates over interchain valence coordinates for two reasons. First, valence coordinates are usually employed to express interactions among atoms which are rather strongly bonded by orbitals which can be described, at least conceptually, in terms of atomic orbitals of the interacting atoms (LCAO-MO's). This concept does not appear to be so precise for expressing the relatively weak interactions between strongly bonded atoms within adjacent spirals in selenium (or between adjacent molecules in a molecular crystal). Second, as has been noted above, the ininterchain valence models have all resorted to rather arbitrary selections among a rather large number of contending valence coordinates. The relative motion coordinates we use avoid the ambiguity. They can be shown to involve linear combinations of all of the interchain valence coordinates.

\section{STRUCTURE}

There exist three crystalline modifications of selenium namely the $\alpha$ and the $\beta$ monoclinic forms and the trigonal torm. The latter is the thermodynamically stable form at all temperatures below the melting point at about $217^{\circ} \mathrm{C}$. The monoclinic forms are thermody namically unstable, and special measures have to be taken in order to produce those forms. The trigonal form is obtained by slowly cooling the melt, whereas the amorphous form is produced if rapid cooling is applied. The structure of the latter has been studied by one of us. ${ }^{11}$ In the temperature range $100-150^{\circ} \mathrm{C}$ the transformation of the thermodynamically unstable forms of selenium to the stable trigonal form takes place with a notable velocity. In the phase diagram in Fig. 1 , the lines for $\alpha$ and $\beta$ monoclinic Se are only qualitative be cause the vapor pressure ${ }^{12}$ is very low at temperatures where they exist for a long time, so an experimental investigation is very difficult.

The monoclinic forms consist of eight membered puckered rings like those known from sulfur, and the binding inside the ring seems to have a strong covalent character, while the inter-ring interactions are weaker. Martin et al. ${ }^{8}$ have made a comparison between the vibration frequencies found in the trigonal form with those calculated for a ring structure as described by Scott et al. ${ }^{13}$

In this paper we only consider the trigonal form, where the atoms are arranged in long spirals with trigonal symmetry. The chains are arranged in a hexagonal pattern, so the Bravais lattice is hexagonal. The crystal belongs to the trigonal system, the space group being either $D_{3}^{4}$ for a left-handed screw axis or $D_{3}^{6}$ for a right-handed screw axis as determined originally by Bradley. ${ }^{14}$ Since the symmetry of the spiral is trigonal the period of the chain is completed for every third atom, so the unit cell contains three atoms. The structure is shown in Fig. 2 and the atomic positions are given in Table I for a right-handed screw axis. The symmetry elements of the $D_{3}^{6}$ space group are shown in Table II.

Selenium has six valence electrons in the $N$ shell. It is assumed that an $s$ orbital and the two $p$ orbitals in the plane of the three atoms in a unit cell form three hybridized orbitals in such a way that two orbitals point towards the two nearest neighbors along the chain giving rise to a strong covalent bonding between the atoms along the chain. These bonding orbitals contain two electrons leaving four electrons in the nonbonding orbitals, the $p$ orbital at right angles to the plane of the three atoms and a hybridized orbital, which is mainly of $s$ type. It is clear that the interaction of the electrons in the nonbonding states are very important for the whole structure and packing of the chains. Even for the chain structure these interactions seem to be very important, because the chain structure is destroyed when the crystal melts, and the melt contains only very small chain fragments and eight membered puckering rings. It

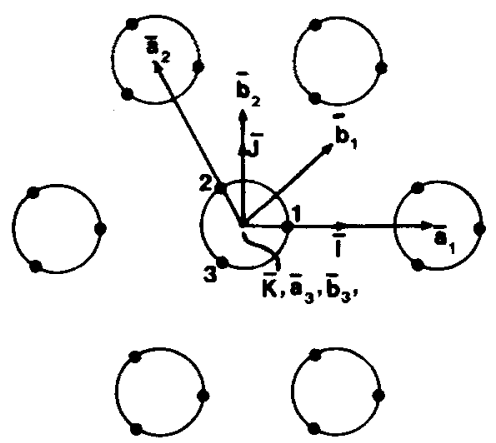

FIG. 2. Structure of trigonal selenium ( $D_{3}^{6}$ space group). The lattice vectors $\left(a_{1}, a_{2}, a_{3}\right)$, the reciprocal lattice vectors $\left(b_{1}\right.$, $\left.b_{2}, b_{3}\right)$, and the central Cartesian coordinates system $(\mathbf{I}, J, K)$ are shown. 
TABLE I. Lattice vectors and atomic positions in trigonal selenium. The data from Ref. 26 have been used.

\begin{tabular}{|c|c|c|c|c|c|}
\hline \multirow{2}{*}{\multicolumn{3}{|c|}{ Room temperature lattice parameters }} & \multicolumn{3}{|c|}{$\begin{array}{l}\text { Atomic positions in the unit cell } \\
\text { represented in the lattice basis } \\
\text { system }\left(\bar{a}_{1}, \bar{a}_{2}, \bar{a}_{3}\right)\end{array}$} \\
\hline & & & Atom No. & Position & Ref. \\
\hline $\begin{array}{c}\left|\bar{a}_{1}\right|=\left|\bar{a}_{2}\right| \\
(\AA)\end{array}$ & $\begin{array}{l}\left|\bar{a}_{3}\right| \\
(\AA)\end{array}$ & Ref. & 1 & 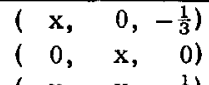 & \\
\hline 4.3662 & 4.9536 & 25 & 3 & $\left(-x,-x, \quad \frac{1}{3}\right)$ & \\
\hline 4.35517 & 4.94945 & 26 & & $x=0.217$ & 26 \\
\hline 4.3712 & 4.9539 & 27 & & $x=0.2254$ & 28 \\
\hline
\end{tabular}

seems also plausible that the mutual repulsion between electrons in the $p$ orbitals of adjacent atoms gives rise to a dihedral torsion force field with a two-fold symmetry. Judging from information from various disulfides and diselenides the barrier is probably of the order of $10 \mathrm{kcal} /$ mole. $^{15}$ Based on these considerations it seems reasonable to use valence-type coordinates from which the symmetrized intrachain coordinates are projected.

It is interesting to note that the trigonal structure may be considered as a degeneration of a hexagonal or even a cubic structure. Martin et al. ${ }^{8}$ show that if the parameter $x=v /\left|a_{1}\right|$, where $v$ is the radius of the chain, becomes equal to $\frac{1}{3}$, the structure ceases to be trigonal and becomes simply hexagonal $(x=0.217$ for trigonal selenium). This means that the nearest-neighbor distance along the chain becomes equal to the nearest-neighbor distance between atoms in different chains. This transition may in fact be realized, when trigonal Se in put under high pressure. ${ }^{16,17}$ When $x=\frac{1}{3}$ and $\left|a_{3}\right| /\left|a_{1}\right|=\sqrt{\frac{3}{2}}$ the structure is cubic.

We have already defined the lattice vectors $a_{1}, a_{2}, a_{3}$ as shown in Fig. 2. In the same figure a Cartesian coordinate system $(I, J, K)$ is defined, which we call the central Cartesian coordinate system. It is used to define absolute positions in the crystal. From Fig. 2 is seen that the two systems are related by

$\left(a_{1}, a_{2}, a_{3}\right)=(I, J, K)\left[\begin{array}{ccc}\left|a_{1}\right| & -\frac{1}{2}\left|a_{1}\right| & 0 \\ 0 & \frac{1}{2} \sqrt{3}\left|a_{1}\right| & 0 \\ 0 & 0 & \left|a_{3}\right|\end{array}\right]$.

The reciprocal lattice vectors $b_{1}, b_{2}, b_{3}$ are defined in the usual way:

$$
b_{i} \cdot a_{j}=2 \pi \delta_{i j},
$$

and given in terms of the central Cartesian coordinate system by

$\left(b_{1}, b_{2}, b_{3}\right)=(I, J, K)\left[\begin{array}{ccc}2 \pi /\left|a_{1}\right| & 0 & 0 \\ 2 \pi / \sqrt{3}\left|a_{1}\right| & 4 \pi / \sqrt{3}\left|a_{1}\right| & 0 \\ 0 & 0 & 2 \pi /\left|a_{3}\right|\end{array}\right]$

The three atoms within a primitive cell possess $C_{2 v}$ symmetry and it will be convenient to use the operations of this group to project the symmetrized coordinates. Therefore, Cartesian axes $1, j, k$ are fixed with- in the primitive cell, with $i$ being the axis of symmetry, $j$ normal to this and in the plane of the atoms, and $k$ is perpendicular to the plane. The unit vectors $\mathbf{i}, j, k$ are related to the I, J, K axes by

$(\mathbf{i}, \mathbf{j}, \mathbf{k})=(\mathbf{I}, \mathbf{J}, \mathbf{K})\left[\begin{array}{ccc}\frac{1}{2} & \frac{3}{4} v / d & c / 2 \sqrt{3} d \\ -\frac{1}{2} \sqrt{3} & \frac{1}{4} \sqrt{3} v / d & c / 6 d \\ 0 & -c / 3 d & \frac{1}{2} \sqrt{3} v / d\end{array}\right]$,

$(\mathbf{I}, \mathbf{j}, \mathbf{k})=(\mathbf{I}, \mathbf{J}, \mathbf{K}) \mathbf{A}$.

The matrix $A$ which appears in Eq. (2.5) is the same as the one which appears in Eq. (2.5b) of I. In Eq. (2.4), $v$ is the spiral radius ( $0.9451 \AA$ in this work), $c=\left|a_{3}\right|$ (4. $9539 \AA$ ), and $d=r \sin \varphi$, where $\varphi$ is half the angle between the bonds from one atom to its nearest neighbors $\left(52.4^{\circ}\right)$ and $r$ is the near-neighbor distance (2.3252 $\AA$ ).

The positions $r_{1}, r_{2}, r_{3}$ of the atoms in the cell are given by

$$
\begin{aligned}
& \left(\mathbf{r}_{1}, \mathbf{r}_{2}, \mathbf{r}_{3}\right)=(\mathbf{i}, \mathbf{j}, \mathbf{k})\left[\begin{array}{ccc}
\frac{1}{2} v & -v & \frac{1}{2} v \\
d & 0 & -d \\
0 & 0 & 0
\end{array}\right], \\
& \left(\mathbf{r}_{1}, \mathbf{r}_{2}, \mathbf{r}_{3}\right)=(\mathbf{I}, \mathbf{J}, \mathbf{K})\left[\begin{array}{ccc}
v & -\frac{1}{2} v & -\frac{1}{2} v \\
0 & \frac{1}{2} \sqrt{3} v & -\frac{1}{2} \sqrt{3} v \\
-\frac{1}{3} c & 0 & \frac{1}{3} c
\end{array}\right] .
\end{aligned}
$$

TABLE II. Symmetry operations of the $D_{3}^{6}$ point group from Kovalev (Ref. 22). The rotation axes are represented in the skew lattice vector coordinate system.

\begin{tabular}{ll}
\hline & $D_{3}^{6}$ space group \\
\hline$\left(h_{1} \mid 0\right)$ & identity \\
$\left(h_{3} \mid \bar{a}_{3} / 3\right)$ & $120^{\circ}$ rotation around $(0,0,1)$ \\
$\left(h_{5} \mid 2 \bar{a}_{3} / 3\right)$ & $240^{\circ}$ rotation around $(0,0,1)$ \\
$\left(h_{7} \mid 0\right)$ & $180^{\circ}$ rotation around $(0,1,0)$ \\
$\left(h_{9} \mid \bar{a}_{3} / 3\right)$ & $180^{\circ}$ rotation around $(1,0,0)$ \\
$\left(h_{11} \mid 2 \bar{a}_{3} / 3\right)$ & $180^{\circ}$ rotation around $(1,1,0)$ \\
\hline
\end{tabular}




\section{COORDINATES FOR THE POTENTIAL ENERGY}

\section{A. Introduction}

We follow the procedures described in I to define a set of symmetrized valence coordinates for expressing the intraspiral interactions, and a set of symmetrized relative motion coordinates for the interchain interactions. It is only necessary to obtain one set of coordinates which are not transformed into each other under the operations of the $D_{3}^{6}$ symmetry group which applies to trigonal selenium. All coordinates equivalent to these may be found by operating on the members of this set with the $D_{3}^{6}$ operations. One of the authors has developed a very general computer program which does this, and also sets up a very general force constant matrices. ${ }^{18}$

In generating the symmetrized coordinates it it useful to employ group theory techniques for projecting displacement patterns having the symmetry of an interacting group of atoms from arbitrary trial patterns. The operators are given $b^{19}$

$$
\hat{P}_{r s}^{(\mu)}=\frac{l_{\mu}}{g} \sum_{i=1}^{g} \chi_{r s}^{(\mu) *}\left(R_{i}\right) \hat{R}_{i},
$$

where $\hat{R}_{i}$ the $i$ th operation of the group, $\chi_{r s}^{(\mu) *}$ is the complex conjugate of the $(r, s)$ element of the representation matrix of $\hat{K}_{i}$ from the $\mu$ th irreducible representation, $l_{\mu}$ is the dimension of the representation, and $g$ is the order of the group.

Due to the covalent character of the bonding along the chain it is natural to project the intrachain coordinates from valence coordinates such as nearest neighbor bond stretch, angle bend between joining near-neighbor bonds and dihedral torsion around a near-neighbor bond.

Symmetric and antisymmetric stretch coordinates like those illustrated in Sec. II of I a re obtained by applying Eq. (3.1) to the operator $b(2,1)$ which projects out the stretch between atoms 2 and 1 in the primitive cell from arbitrary displacements of the atoms. It is sufficient to use the representation table for the $C_{2}$ group to accomplish this.

The projection operator $b(2,1)$ is expressed using components of a unit vector along the line from atom 2 to atom 1 according to the usual Wilson method. ${ }^{20}$ Fol lowing $I$ these components are written as a row vector

$$
\hat{\mathrm{t}}^{T}(2,1)=[\cos \varphi, \sin \varphi, 0],
$$

where $\dot{\varphi}$ is half the angle between the bonds from atom 2 to atom 1 , and from 2 to 3 . Then $b(2,1)$ is given by ${ }^{20}$

$$
\begin{aligned}
& \left(\begin{array}{lll}
0 & 0 & 0 \\
1
\end{array}\right)\left(\begin{array}{lll}
0 & 0 & 0 \\
2
\end{array}\right)\left(\begin{array}{lll}
0 & 0 & 0 \\
3
\end{array}\right) \\
& \mathbf{b}(2,1)=\left[\hat{\mathrm{t}}^{T}(2,1), \quad-\hat{\mathbf{t}}^{T}(2,1),\right.
\end{aligned}
$$

where the designations $\left({ }^{l_{1}, l_{2}, t_{3}}\right)$ denote the cell indices (all $l_{1}=0$ for the origin cell) and the atom $\nu$ involved. The 0 in Eq. (3. 3) denotes a row of three zero elements.

The operation of $180^{\circ}$ rotation about $a_{2}$ converts $\hat{\mathrm{t}}^{T}(2,1)$ into $\hat{\mathrm{t}}^{T}(2,3)$ which is

$$
\hat{\mathrm{t}}^{T}(2,3)=[\cos \varphi,-\sin \phi, 0] \text {. }
$$

Application of Eq. (3.1) using the $A$ and $B$ representa tions of $C_{2}$ leads to the operators $b_{1}$ and $b_{2}$ which project the symmetric and antisymmetric stretches

$$
\begin{gathered}
\left(\begin{array}{lll}
0 & 0 & 0 \\
1
\end{array}\right),\left(\begin{array}{lll}
0 & 0 & 0 \\
2
\end{array}\right),\left(\begin{array}{lll}
0 & 0 & 0 \\
3
\end{array}\right) \\
\mathrm{b}_{1}=\left[\begin{array}{l}
\hat{\mathrm{t}}^{T}(2,1),-[\hat{\mathrm{t}}(2,1)+\hat{\mathrm{t}}(2,3)]^{T}, \\
\left.\hat{\mathrm{t}}^{T}(2,3)\right], \\
\left(\begin{array}{lll}
0 & 0 & 0 \\
1
\end{array}\right)\left(\begin{array}{lll}
0 & 0 & 0 \\
2
\end{array}\right)\left(\begin{array}{lll}
0 & 0 & 0 \\
3
\end{array}\right)
\end{array}\right) \\
\mathrm{b}_{2}=\left[\hat{\mathrm{t}}^{T}(2,1),-[\hat{\mathrm{t}}(2,1)-\hat{\mathrm{t}}(2,3)]^{T},-\hat{\mathrm{t}}^{T}(2,3)\right] .
\end{gathered}
$$

The factor $1 / g=\frac{1}{4}$ in Eq. (3.1) has been omitted in these equations.

The operator for the angle bend coordinate is given in terms of unit vectors which are in the plane of the three atoms and are normal to the 2-1 and 2-3 bonds. They are directed outward from atoms 1 and 3 so as to open the angle between the bonds. We will denote the row vectors made up of the components of these unit vectors by $\hat{\mathrm{t}}^{T}(\psi, 1)$ and $\hat{\mathrm{t}}^{T}(\psi, 3)$. Then ${ }^{20}$

$\hat{\mathbf{t}}^{T}(\psi, 1)=[-\sin \varphi, \cos \varphi, 0], \quad \hat{\mathrm{t}}^{T}(\psi, 3)=[-\sin \varphi,-\cos \varphi, 0]$.

The projector for the angle bend, which belongs to the $A_{1}$ representation of $C_{2 v}$ (or the $A$ representation of $C_{2}$ ) is

$$
\begin{aligned}
& \left(\begin{array}{ccc}
0 & 0 & 0 \\
& 1
\end{array}\right)\left(\left(\begin{array}{lll}
0 & 0 & 0 \\
& 2
\end{array}\right)\left(\begin{array}{lll}
0 & 0 & 0 \\
& 3
\end{array}\right)\right. \\
& b_{3}=(1 / \gamma)\left[\hat{\mathfrak{t}}^{T}(\psi, 1),-\left[\hat{\mathbf{t}}^{T}(\psi, 1)+\hat{\mathfrak{t}}^{T}(\psi, 3), \hat{\mathrm{t}}^{T}(\psi, 3)\right] .\right.
\end{aligned}
$$

The symmetrized dihedral torsion projectors are obtained from the projector $B_{\tau}(1-2)$ for a conventional torsion about the $1-2$ bond. The notation $B_{r}(1-2)$ is used because it is easiest to obtain this projector directly in terms of components along I, J, K [Eq. (2.8) of I]. The elements can be obtained from components of vectors $\mathbf{B}\left({ }^{0}{ }_{3}^{0}-1\right)$ and $\mathbf{B}\left(\left(^{0}{ }_{3}^{0}\right)\right.$ defined as by Wilson ${ }^{20}$ for the end atoms, and using the equations for the interior atom vectors as corrected by Herman. ${ }^{21}$ The $\mathbf{B}\left({ }^{0}{ }_{3}^{0}-1\right)$ and $\mathbf{B}\left(\begin{array}{lll}0 & 0 & 0\end{array}{ }^{0}\right)$ are

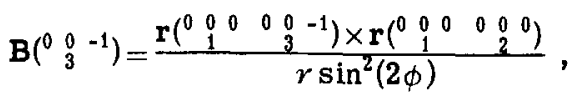

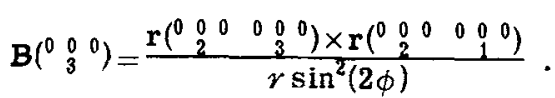

The $\mathbf{B}\left(\begin{array}{lll}0 & 0 & 0 \\ 1\end{array}\right)$ and $\mathbf{B}\left(\begin{array}{lll}0 & 0 & 0 \\ 2\end{array}\right)$ may be written ${ }^{21}$

$$
\begin{aligned}
\mathrm{B}\left(\begin{array}{lll}
0 & 0 & 0 \\
& 1
\end{array}\right)= & -\left\{\mathrm{B}\left(\begin{array}{ccc}
0 & 0 & -1 \\
3
\end{array}\right)\right. \\
& \left.+\cos ^{2} \phi\left[\mathrm{B}\left(\begin{array}{lll}
0 & 0 & 0 \\
& 3
\end{array}\right)-\mathrm{B}\left(\begin{array}{lll}
0 & 0 & -1 \\
3
\end{array}\right)\right]\right\},
\end{aligned}
$$




$$
\begin{aligned}
\mathbf{B}\left(\begin{array}{lll}
0 & 0 & 0 \\
& 2
\end{array}\right)= & -\left\{\mathbf{B}\left(\begin{array}{lll}
0 & 0 & 0 \\
3
\end{array}\right)\right. \\
& \left.+\cos ^{2} \phi\left[\mathbf{B}\left(\begin{array}{lll}
0 & 0 & -1 \\
& 3
\end{array}\right)-\mathbf{B}\left(\begin{array}{lll}
0 & 0 & 0 \\
& 3
\end{array}\right)\right]\right\} .
\end{aligned}
$$

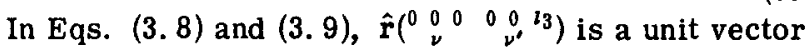
from atom $\nu$ in the origin cell toward atom $\nu^{\prime}$ in the cell at $0,0, l_{3}$, where $l_{3}=-1$ or 0 .

The projector $B_{\tau}(1-2)$ is then written using the components of the $\mathrm{B}\left({ }^{0}{ }_{\nu}^{0}{ }^{t_{3}}\right)$ to form the row vectors in the following projector:

$$
\begin{aligned}
\mathbf{B}_{\tau}(1-2)= & {\left[0, \mathbf{B}\left(\begin{array}{lll}
0 & 0 & 0 \\
& 3
\end{array}\right), \mathrm{B}\left(\begin{array}{lll}
0 & 0 & 0 \\
& 2
\end{array}\right), \mathrm{B}\left(\begin{array}{lll}
0 & 0 & 0 \\
& 1
\end{array}\right),\right.} \\
& \left.\mathbf{B}\left(\begin{array}{rrr}
0 & 0 & -1
\end{array}\right)\right],
\end{aligned}
$$

where 0 denotes a row of three zeros and

$$
\begin{aligned}
& \mathbf{B}\left(\begin{array}{lll}
0 & 0 & 0 \\
& 3
\end{array}\right)=\frac{1}{D}\left[\frac{\sqrt{3}}{2} \frac{c}{v}, \frac{1}{2} \frac{c}{v}, \frac{3 \sqrt{3}}{2}\right], \\
& \mathbf{B}\left(\begin{array}{lll}
0 & 0 & -1 \\
& 3
\end{array}\right)=\frac{1}{D}\left[0, \frac{c}{v}, \frac{-3 \sqrt{3}}{2}\right],
\end{aligned}
$$

where $c=\left|a_{3}\right|(4.9539 \AA), v$ is the spiral radius $(0.9451$ $\AA$ ), and

$$
D=r \sin ^{2}(2 \phi)\left[3+(c / 3 v)^{2}\right]=13.2 \AA .
$$

When the displacement pattern defined by $B_{T}(1-2)$ is operated on by a $180^{\circ}$ rotation about $a_{2}$, a projector $B_{\tau}(2-3)$ for a torsion about the bond between atoms 2 and 3 is obtained. Application of Eq. (3.1) using the $C_{2}$ group leads to projectors $B_{4}$ and $B_{5}$ for the symmetric and antisymmetric dihedral torsions given by

$$
\begin{aligned}
& \mathrm{B}_{4}=\left[\mathrm{B}_{4}\left(\begin{array}{lll}
0 & 0 & 1 \\
& 1 &
\end{array}\right), \mathrm{B}_{4}\left(\begin{array}{lll}
0 & 0 & 0 \\
& 3 &
\end{array}\right), \mathrm{B}_{4}\left(\begin{array}{lll}
0 & 0 & 0 \\
& 2 &
\end{array}\right), \mathrm{B}_{4}\left(\begin{array}{lll}
0 & 0 & 0 \\
& 1
\end{array}\right), \mathrm{B}_{4}\left(\begin{array}{ccc}
0 & 0 & -1 \\
3 &
\end{array}\right)\right] \\
& \mathbf{B}_{5}=\left[\mathbf{B}_{5}\left(\begin{array}{lll}
0 & 0 & 1 \\
& 1 &
\end{array}\right), \mathbf{B}_{5}\left(\begin{array}{lll}
0 & 0 & 0 \\
& 3 &
\end{array}\right), \mathbf{B}_{5}\left(\begin{array}{lll}
0 & 0 & 0 \\
& 2
\end{array}\right), \mathbf{B}_{5}\left(\begin{array}{lll}
0 & 0 & 0 \\
& 1
\end{array}\right), \mathbf{B}_{5}\left(\begin{array}{ccc}
0 & 0 & -1 \\
& 3
\end{array}\right)\right] \text {; } \\
& \mathbf{B}_{4}\left(\begin{array}{lll}
0 & 0 & 1 \\
& 1
\end{array}\right)=\frac{1}{D}\left[-\frac{c}{v} \frac{\sqrt{3}}{2}, \frac{1}{2} \frac{c}{v}, \frac{3 \sqrt{3}}{2}\right] \text {, } \\
& \mathrm{B}_{4}\left(\begin{array}{lll}
0 & 0 & 0 \\
3
\end{array}\right)=\frac{1}{D}\left[\frac{\sqrt{3} c}{v}, \frac{c \cos ^{2} \phi}{v}, 3 \sqrt{3} \cos ^{2} \phi\right] \text {, } \\
& \mathrm{B}_{4}\left(\begin{array}{lll}
0 & 0 & 0 \\
2
\end{array}\right)=\frac{1}{D}\left[\frac{\sqrt{3}}{2} \frac{c \cos ^{2} \phi}{v},-\frac{3}{2} \frac{c \cos ^{2} \phi}{v}, 0\right] \text {, } \\
& \mathrm{B}_{4}\left(\begin{array}{ccc}
0 & 0 & 0 \\
& 1
\end{array}\right)=\frac{1}{D}\left[-\frac{\sqrt{3}}{2} \frac{c\left(1+\cos ^{2} \phi_{1}\right)}{v},-\frac{c}{v}\left(\frac{3-\cos ^{2} \phi}{2}\right),-3 \sqrt{3} \cos ^{2} \phi\right] \text {; } \\
& \mathbf{B}_{4}\left(\begin{array}{ccc}
0 & 0 & -1 \\
& 3
\end{array}\right)=\frac{1}{D}\left[0, \frac{c}{v},-\frac{3 \sqrt{3}}{2}\right], \\
& \mathrm{B}_{5}\left(\begin{array}{lll}
0 & 0 & 1 \\
& 1
\end{array}\right)=\frac{1}{D}\left[\frac{\sqrt{3}}{2} \frac{c}{v},-\frac{1}{2} \frac{c}{v},-\frac{3 \sqrt{3}}{2}\right] \text {, } \\
& \mathbf{B}_{5}\left(\begin{array}{lll}
0 & 0 & 0 \\
& 3
\end{array}\right)=\frac{1}{D}\left[0, \frac{c\left(1-\cos ^{2} \phi\right)}{v}, 3 \sqrt{3}\left(1-\cos ^{2} \phi\right)\right] \text {, } \\
& \mathbf{B}_{5}\left(\begin{array}{lll}
0 & 0 & 0 \\
& 2
\end{array}\right)=\frac{1}{D}\left[-\sqrt{3}\left(1-\frac{\cos ^{2} \phi}{2}\right) \frac{c}{v},-\left(1-\frac{\cos ^{2} \phi}{2}\right) \frac{c}{v},-3 \sqrt{3}\left(1-2 \cos ^{2} \phi\right)\right] \text {, } \\
& \mathrm{B}_{5}\left(\begin{array}{lll}
0 & 0 & 0 \\
& 1
\end{array}\right)=\frac{1}{D}\left[\frac{\sqrt{3}}{2} \frac{\left(1-\cos ^{2} \phi\right) c}{v},-\frac{1}{2} \frac{c\left(1-\cos ^{2} \phi\right)}{v}, 3 \sqrt{3}\left(1-\cos ^{2} \phi\right)\right] \text {, } \\
& \mathrm{B}_{5}\left(\begin{array}{lll}
0 & 0 & -1 \\
& 3 &
\end{array}\right)=\frac{1}{D}\left[0, \frac{c}{v},-\frac{3 \sqrt{3}}{2}\right] \text {. }
\end{aligned}
$$


As in Eq. (3.6) the factor $1 / g=\frac{1}{4}$ is omitted in obtaining Eqs. (3.17) and (3.18).

\section{B. Intercell relative motion coordinates}

The basis set of relative motion coordinates for expressing the interspiral interactions are given in terms of relative translations and relative rotations of the atoms in the origin cell and the cell at $(0,1,0)$. The axes within each group are the same as those used for coordinates $1-3$ as expressed in Eq. (2.4). Axes parallel to these with origin midway between the two groups along $a_{2}$ are used to define the matrices $R(1)$ and $\mathbf{R}(2)$ of Eq. (3.23) of $I$, which are used in Eqs. (3.26) and (3.27) of $I$ to define the relative translation coordinates which are generated from displacements accompanying pure rotations.

There are five relative rotation coordinates. Two are given by Eqs. (3. 31) and (3.32) of I with $\hat{\alpha}^{T}=[0,1,0]$ and $[0,0,1]$. They correspond to cases where both groups rotate in the same sense about $j$ and $k$, respectively.
Two other coordinates are counter rotation about these axes and the fifth one is a counter rotation about 1 .

The projectors for these coordinates are defined from Eqs. (3.16) and (3.31) of I using the atom positions in the $\mathbf{i}, \mathbf{j}, \mathrm{k}$ systems given by Eq. (2.4). Thus

$$
\begin{aligned}
& \mathbf{r}_{1}(1)=\mathbf{r}_{1}(2)=\left[\begin{array}{ccc}
0 & 0 & -d \\
0 & 0 & \frac{1}{2} v \\
d & -\frac{1}{2} v & 0
\end{array}\right], \\
& \mathbf{r}_{2}(1)=\mathbf{r}_{2}(2)=\left[\begin{array}{ccc}
0 & 0 & 0 \\
0 & 0 & -v \\
0 & v & 0
\end{array}\right], \\
& \mathbf{r}_{3}(1)=\mathbf{r}_{3}(2)=\left[\begin{array}{ccc}
0 & 0 & d \\
0 & 0 & \frac{1}{2} v \\
-d & -\frac{1}{2} v & 0
\end{array}\right] .
\end{aligned}
$$

Equation (3.32) of I with $\hat{\alpha}^{T}=[0,1,0]$ leads to the projector

$$
\begin{aligned}
& \left(\begin{array}{lll}
0 & 0 & 0 \\
1
\end{array}\right)\left(\begin{array}{lll}
0 & 0 & 0 \\
2 &
\end{array}\right)\left(\begin{array}{lll}
0 & 0 & 0 \\
3
\end{array}\right)\left(\begin{array}{lll}
0 & 1 & 0 \\
1
\end{array}\right)\left(\begin{array}{lll}
0 & 1 & 0 \\
2
\end{array}\right)\left(\begin{array}{lll}
0 & 1 & 0 \\
3
\end{array}\right) \\
& \mathrm{b}_{6} \equiv \mathrm{b}_{r y}=(1 / 3 v)\left[0,0,-\frac{1}{2}, \quad 0,0,1, \quad 0,0,-\frac{1}{2}, 0,0,-\frac{1}{2}, 0,0,1,0,0,-\frac{1}{2}\right],
\end{aligned}
$$

The counter rotation about $j$ as defined using Eq. (3.16) of $I$ is

$$
\left(\begin{array}{lll}
0 & 0 & 0 \\
& 1 &
\end{array}\right)\left(\begin{array}{lll}
0 & 0 & 0 \\
& 2 &
\end{array}\right)\left(\begin{array}{lll}
0 & 0 & 0 \\
& 3 &
\end{array}\right)\left(\begin{array}{lll}
0 & 1 & 0 \\
& 1 &
\end{array}\right)\left(\begin{array}{lll}
0 & 1 & 0 \\
2 &
\end{array}\right)\left(\begin{array}{lll}
0 & 1 & 0 \\
& 3 &
\end{array}\right)
$$

$\mathbf{b}_{7}=(2 / 3 v)\left\lfloor 0,0,-\frac{1}{2}, \quad 0,0,1, \quad 0,0,-\frac{1}{2}, \quad 0,0, \frac{1}{2}, \quad 0,0,-1, \quad 0,0, \frac{1}{2}\right]$.

Similarly the projectors for the parallel and antiparallel rotation about $\mathbf{k}$ are

$$
\begin{aligned}
& \left(\begin{array}{lll}
0 & 0 & 0 \\
& 1 &
\end{array}\right)\left(\begin{array}{lll}
0 & 0 & 0 \\
& 2 &
\end{array}\right)\left(\begin{array}{lll}
0 & 0 & 0 \\
& 3 &
\end{array}\right)\left(\begin{array}{lll}
0 & 1 & 0 \\
& 1 &
\end{array}\right)\left(\begin{array}{lll}
0 & 1 & 0 \\
& 2 &
\end{array}\right)\left(\begin{array}{lll}
0 & 1 & 0 \\
& 3 &
\end{array}\right) \\
& \mathrm{b}_{8}=x\left[\frac{-d}{v}, \frac{1}{2}, 0, \quad 0,-1,0, \quad \frac{d}{v}, \frac{1}{2}, 0, \frac{-d}{v}, \frac{1}{2}, 0, \quad 0,-1,0, \quad \frac{d}{v}, \frac{1}{2}, 0\right] \text {, } \\
& \left(\begin{array}{lll}
0 & 0 & 0 \\
& 1 &
\end{array}\right)\left(\begin{array}{lll}
0 & 0 & 0 \\
& 2 &
\end{array}\right)\left(\begin{array}{lll}
0 & 0 & 0 \\
& 3 &
\end{array}\right)\left(\begin{array}{lll}
0 & 1 & 0 \\
& 1 &
\end{array}\right)\left(\begin{array}{lll}
0 & 1 & 0 \\
& 2 &
\end{array}\right)\left(\begin{array}{lll}
0 & 1 & 0 \\
& 3 &
\end{array}\right) \\
& \mathrm{b}_{9}=2 x\left[\frac{-d}{v}, \frac{1}{2}, 0, \quad 0,-1,0, \quad \frac{d}{v}, \frac{1}{2}, 0, \quad \frac{d}{v}, \frac{-1}{2}, 0, \quad 0,1,0, \frac{-d}{v}, \frac{-1}{2}, 0\right] \text {, } \\
& \text { where } x=\left[3 v\left(1+4 d^{2} / 3 v^{2}\right)\right]^{-1} \text {. }
\end{aligned}
$$

Finally, the projector for the counter rotation about 1 is given by using Eq. (3. 16) of $I$ with $\hat{\theta}^{T}=[1,0,0]$,

$$
\left(\begin{array}{lll}
0 & 0 & 0 \\
& 1 &
\end{array}\right)\left(\begin{array}{lll}
0 & 0 & 0 \\
& 2 &
\end{array}\right)\left(\begin{array}{lll}
0 & 0 & 0 \\
& 3 &
\end{array}\right)\left(\begin{array}{lll}
0 & 1 & 0 \\
& 1 &
\end{array}\right)\left(\begin{array}{lll}
0 & 1 & 0 \\
& 2
\end{array}\right)\left(\begin{array}{lll}
0 & 1 & 0
\end{array}\right)
$$

$b_{10}=(1 / 2 d)[0,0,1, \quad 0,0,0, \quad 0,0,-1, \quad 0,0,-1, \quad 0,0,0, \quad 0,0,1]$.

There are three relative translation coordinates. One is a counter translation along $\mathbf{i}$ and its projector is given by Eq. (3. 7 ) of $I$ using $\hat{t}^{T}=(1,0,0)$. Thus 


$$
\begin{aligned}
& \left(\begin{array}{lll}
0 & 0 & 0 \\
1 & 1
\end{array}\right)\left(\begin{array}{lll}
0 & 0 & 0 \\
2 &
\end{array}\right)\left(\begin{array}{lll}
0 & 0 & 0 \\
3 &
\end{array}\right)\left(\begin{array}{lll}
0 & 1 & 0 \\
1
\end{array}\right)\left(\begin{array}{lll}
0 & 1 & 0 \\
2
\end{array}\right)\left(\begin{array}{lll}
0 & 1 & 0 \\
& 3
\end{array}\right) \\
& \mathbf{b}_{11}=\frac{1}{3}[1,0,0,-1,0,0, \quad 1,0,0, \quad-1,0,0, \quad 1,0,0,-1,0,0] .
\end{aligned}
$$

The final two relative translations are obtained from Eqs. (3.26) and (3.27) of I with $\hat{\alpha}^{T}=[0,1,0]$ and $[0,0,1]$.

They are defiried using $R(1)$ and $R(2)$ from Eq. (3.23) of $I$ with

$$
\begin{aligned}
& x(1)=\frac{1}{2} a_{2}, x(2)=-\frac{1}{2} a_{2}, \\
& y(1)=y(2)=z(1)=z(2)=0 .
\end{aligned}
$$

Then

$$
\mathbf{R}(1)=\frac{1}{2} a_{2}\left[\begin{array}{ccc}
0 & 0 & 0 \\
0 & 0 & 1 \\
0 & -1 & 0
\end{array}\right], \quad \mathbf{R}(2)=-\mathbf{R}(1),
$$

giving

$$
\begin{aligned}
& \left(\begin{array}{lll}
0 & 0 & 0 \\
& 1
\end{array}\right)\left(\begin{array}{lll}
0 & 0 & 0 \\
& 2 &
\end{array}\right)\left(\begin{array}{lll}
0 & 0 & 0 \\
& 3 &
\end{array}\right)\left(\begin{array}{lll}
0 & 1 & 0 \\
& 1 &
\end{array}\right)\left(\begin{array}{lll}
0 & 1 & 0 \\
& 2 &
\end{array}\right)\left(\begin{array}{lll}
0 & 1 & 0 \\
& 3
\end{array}\right) \\
& \mathrm{b}_{12}=\mathrm{b}_{R_{y}}=\left(-1 / 3 a_{2}\right)[0,0,1, \quad 0,0,1, \quad 0,0,1, \quad 0,0,-1, \quad 0,0,-1, \quad 0,0,-1], \\
& \left(\begin{array}{lll}
0 & 0 & 0 \\
& 1
\end{array}\right)\left(\begin{array}{lll}
0 & 0 & 0 \\
& 2 &
\end{array}\right)\left(\begin{array}{lll}
0 & 0 & 0 \\
3
\end{array}\right)\left(\begin{array}{lll}
0 & 1 & 0 \\
1 & 1
\end{array}\right)\left(\begin{array}{lll}
0 & 1 & 0 \\
& 2
\end{array}\right)\left(\begin{array}{lll}
0 & 1 & 0 \\
& 3
\end{array}\right) \\
& \mathbf{b}_{13}=b_{R z}=\left(1 / 3 a_{2}\right)[0,1,0, \quad 0,1,0, \quad 0,1,0, \quad 0,-1,0, \quad 0,-1,0, \quad 0,-1,0] \text {. }
\end{aligned}
$$

Tables III and IV summarize the coordinates and give their symmetries and the force constants used in calculating the dispersion curves in Figs. 4 and 5 . The interaction constants $f_{6,12}$ and $f_{8,13}$ are calculated from Eq. (4.2) of $I$ and ensure that the potential energy is rotationally invariant.

The projector operators for coordinates $1-3$ and 6-13 must be operated on by the matrix $\mathbf{A}^{T}$ obtained from Eq. (2.4) and used as in Eq. (2.8) of $I$.

The coordinates in Tables III and IV form a nonequivalent set. Coordinates equivalent to the se take the same force constants and were obtained using a computer code ${ }^{18}$ which carries out the necessary transformations using the operations of $D_{3}^{6}$ appropriate to trigonal selenium. This provided the full set of coordinates needed to define the potential.

\section{SECULAR EQUATION}

Following I the secular equation is expressed in terms of mass adjusted coordinates suited to simplifying the kinetic energy. These coordinates are components of mass adjusted basis vectors which transform according to the group of the wave vector. They can be derived by applying Eq. (3.1) to selected displacement patterns of the atoms in the origin cell. Since trigonal selenium is a nonsymmorphic crystal it is necessary to use multiplier representations of the space group rather than ordinary point group representation. ${ }^{19}$ The relevant basis vectors $\hat{L}$ (in the notation of $I$ ) are given in Tables VI-DX and the labeling of the points in the Brillouin zone are as given in Fig. 3 where $\left(\zeta_{1}, \zeta_{2}, \zeta_{3}\right)$ are along the reciprocal vectors $b_{1}, b_{2}$, and $b_{3}$. Table $V$ gives the group of the wave vector associated with Tables VIIX.

\section{EXPERIMENTAL RESULTS}

The first measurements of the phonon dispersion relations for trigonal selenium were reported by Hamilton et al. ${ }^{1}$ in 1974. They performed measurements in the acoustical regime at $300 \mathrm{~K}$ in the directions

$$
\Gamma \rightarrow A-H-K \rightarrow \Gamma-M-L \rightarrow A,
$$

and at $77 \mathrm{~K}$ in the acoustical and lower optical range in the directions

$$
\Gamma \rightarrow A, \quad \Gamma-K, \quad \text { and } \Gamma \rightarrow M .
$$

The attempts to study the upper optical modes failed, however. Later the high optical modes were measured by Teuchert et al. , ${ }^{2}$ where also the phonon dispersion relations in the acoustical and middle optical range were included. The latter were, within the experimen-

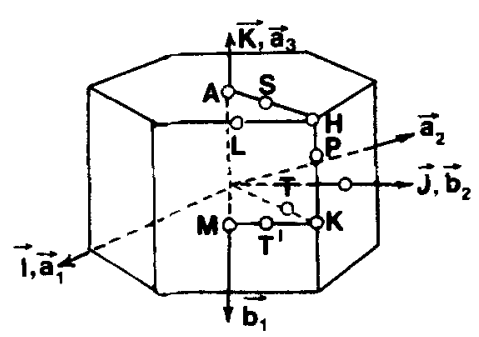

FIG. 3. Labeling of various directions in the Brillouin zone after Koster (Ref. 23). 
TABLE III. Intrachain coordinates, their symmetries and force constants.

\begin{tabular}{|c|c|c|c|c|}
\hline \multicolumn{4}{|c|}{ Intrachain coordinates } & \multirow{2}{*}{$\begin{array}{l}\text { Force } \\
\text { constant } \\
\text { (mdyn } / \AA)\end{array}$} \\
\hline $\begin{array}{l}\text { Coordinate } \\
\text { No. }\end{array}$ & Description & $\begin{array}{l}C_{2 v} \\
\text { symmetry }\end{array}$ & $\begin{array}{l}C_{2} \\
\text { symmetry }\end{array}$ & \\
\hline 1 & $\begin{array}{l}\text { Symmetrized nearest- } \\
\text { neighbor bond stretch } \\
\text { [Eq. }(3.3)]\end{array}$ & $A_{1}$ & $\boldsymbol{A}$ & 0.660 \\
\hline 2 & $\begin{array}{l}\text { Antisymmetrized nearest- } \\
\text { neighbor bond stretch } \\
\text { [Eq. (3.6)] }\end{array}$ & $B_{1}$ & $B$ & 0.152 \\
\hline 3 & $\begin{array}{l}\text { Angle bend between adjacent } \\
\text { nearest neighbor } \\
\text { [Eq. (3.7)] }\end{array}$ & $A_{1}$ & $A$ & 0.847 \\
\hline 4 & $\begin{array}{l}\text { Symmetrized dihedral } \\
\text { torsion [Eq. }(3.15)]\end{array}$ & $\cdots$ & $A$ & 0.0377 \\
\hline \multirow[t]{3}{*}{5} & $\begin{array}{l}\text { Antisymmetrized dihedral } \\
\text { torsion [Eq. }(3.16)]\end{array}$ & $\cdots$ & $B$ & 0.0663 \\
\hline & Cross terms: & & & \\
\hline & $\begin{array}{l}f_{1,3} \\
f_{1,4} \\
f_{2,5} \\
f_{3,4}\end{array}$ & $\begin{array}{l}A_{1} \\
\cdots \\
\cdots \\
\cdots\end{array}$ & $\begin{array}{l}A \\
A \\
B \\
A\end{array}$ & $\begin{array}{l}0.435 \\
0.0641 \\
0.0488 \\
0.111\end{array}$ \\
\hline
\end{tabular}

tal error, in agreement with Hamilton's results. At Grenoble $^{2}$ the measurements were performed at $300 \mathrm{~K}$ for the directions

$$
\Gamma \rightarrow A \rightarrow H \rightarrow K \rightarrow \Gamma \rightarrow M \rightarrow K .
$$

Comparison between the lower optical modes at 77 and $300 \mathrm{~K}$ indicates that the frequencies are increased by $(5-10) \%$ due to anharmonic effects, when the temperature is lowered from 300 to $77 \mathrm{~K}$. A remarkable result is the strong dispersion and splitting of the upper optical modes, especially in the $a_{3}$ direction.

At the center of the Brillouin zone, ir, and Raman data are given by Ref. 4 and elasticity data and sound velocities are given by Ref. 28 .

\section{DETERMINATION OF FORCE CONSTANTS}

Since there does not exist a computation of the potential energy surface for a selenium crystal, which would enable a direct calculation of the force constants associated with the various coordinates, we have determined the force constants from a least-squares fit to the observed frequencies of Teuchert et al. ${ }^{2}$

From the definition of the coordinates it is clear that the intrachain coordinates determine the dispersion relations in directions along the screw axes, whereas interchain coordinates determine the dispersion in direc tions at right angles to the screw axis. This, in fact, greatly simplifies the determination of the force con-

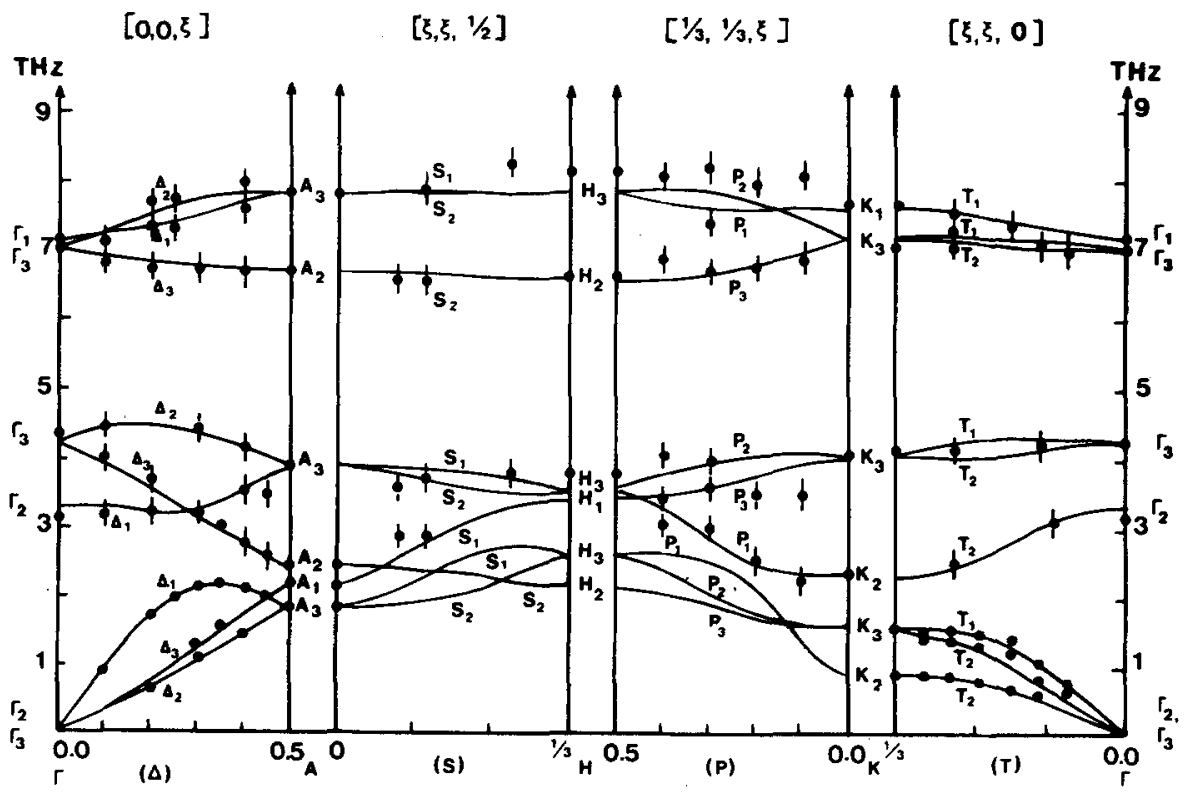

FIG. 4. Calculated phonon dispersion relations for selenium. Experimental points are shown by dots. 
TABLE IV. Interchain coordinates, their symmetries and force constants.

\begin{tabular}{|c|c|c|c|c|}
\hline \multicolumn{5}{|c|}{ Interchain coordinates } \\
\hline \multirow{2}{*}{$\begin{array}{l}\text { Coordinate } \\
\text { No. }\end{array}$} & \multirow[b]{2}{*}{ Description } & \multicolumn{2}{|c|}{ Symmetry } & \multirow{2}{*}{$\begin{array}{l}\text { Force } \\
\text { constant } \\
\text { (mdyn/ } / \AA) \\
\end{array}$} \\
\hline & & $C_{2 v}$ & $c_{2}$ & \\
\hline 6 & $\begin{array}{l}\text { Rigid rotation of unit cell } \\
\text { atoms in adjacent chains } \\
\text { around } j \text { in the same sense } \\
{[\text { Eq. }(3.20)]}\end{array}$ & $B_{2}$ & $B$ & 0.496 \\
\hline 7 & $\begin{array}{l}\text { Rigid rotation of unit cell } \\
\text { atoms in adjacent chains } \\
\text { around } \mathrm{j} \text { in the opposite sense } \\
\text { [Eq. }(3.21)]\end{array}$ & $B_{2}$ & $B$ & 0.036 \\
\hline 8 & $\begin{array}{l}\text { Rigid rotation of unit cell atoms } \\
\text { in adjacent chains around } \mathrm{k} \\
\text { in the same sense [Eq. }(3.22)]\end{array}$ & $B_{1}$ & $B$ & 0.620 \\
\hline 9 & $\begin{array}{l}\text { Rigid rotation of unit cell atoms } \\
\text { in adjacent chains around } \mathrm{k} \text { in } \\
\text { opposite sense [Eq. }(3.23)]\end{array}$ & $B_{1}$ & $B$ & 0.0451 \\
\hline 10 & $\begin{array}{l}\text { Rigid rotation of unit cell atoms } \\
\text { in adjacent chains around } l \text { in } \\
\text { opposite sense [Eq. }(3.24)]\end{array}$ & $A_{2}$ & $A$ & 0.534 \\
\hline 11 & $\begin{array}{l}\text { Relative translation of unit cell } \\
\text { atoms in adjacent chains along } \\
1 \text { [Eq. }(3.25)]\end{array}$ & $A_{1}$ & $A$ & 0.059 \\
\hline 12 & $\begin{array}{l}\text { Relative translation of unit cell } \\
\text { atoms in adjacent chains along } \\
\mathbf{k}[\mathrm{Eq} .(3.26)]\end{array}$ & $B_{2}$ & $B$ & 0.524 \\
\hline \multirow[t]{3}{*}{13} & $\begin{array}{l}\text { Relative translation of unit } \\
\text { cell atoms in adjacent chains } \\
\text { along } J[E q .(3.27)]\end{array}$ & $B_{1}$ & $B$ & 0.171 \\
\hline & Cross terms: & & & \\
\hline & $\begin{array}{l}f_{6,12}[\mathrm{Eq} .(4.2) \text { of } \mathrm{I}] \\
f_{8,13}[\mathrm{Eq} .(4.2) \text { of } \mathrm{I}]\end{array}$ & $\begin{array}{l}B_{2} \\
B_{1}\end{array}$ & $\begin{array}{l}B \\
B\end{array}$ & $\begin{array}{l}-0.510 \\
-0.396\end{array}$ \\
\hline
\end{tabular}

stants. The frequency data in the $\Gamma \rightarrow A$ direction have been used to evaluate the force constants associated with the intrachain coordinates, and frequency data in the $\Gamma \rightarrow K$ direction have been used for the determination of force constants for the interchain coordinates. The symmetry properties of the coordinates also provide the basis for another simpliciation, as already mentioned. We only consider couplings between coor dinates of like symmetry according to the local $C_{2 v}$ (or $C_{2}$ ) symmetry. Still, there is a limitation to that rule. It is simple to show that once the constraint equations have been set up to insure rotational invariance of the potential energy, any additional couplings between coordinates both of which are noninvariant, are not admissible. If such interactions are introduced the invariance is destroyed. The results in Tables III and IV reflect these rules.

The use of symmetry coordinates (Tables VI, VII, VIII, and IX) to block the dynamical matrix is also of great help in the determination of force constants. At $\Gamma$, for example, the nonzero $\Gamma_{2}$ frequency is determined by only two force constants $f_{6,6}$ and $f_{8,8}$. At $A$ it was found that only coordinate 3 made a contribution to the

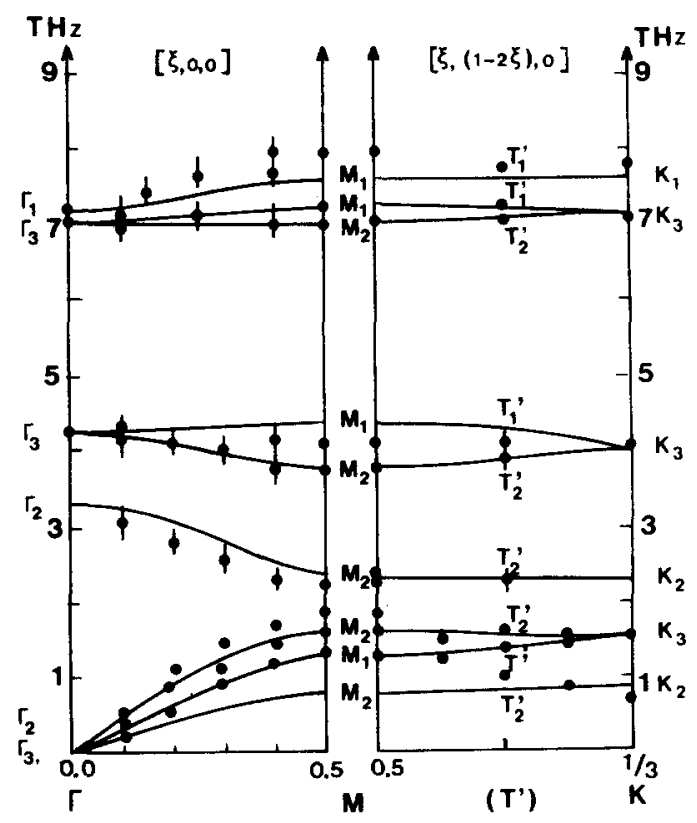

FIG. 5, Calculated phonon dispersion relations for selenium. Experimental points are shown by dots. 
TABLE V. Group of various wave vectors $k$ for trigonal selenium. The numerals $1,3,5,7$, 9,11 in the top rows signify the operations $h_{1}, h_{3}, h_{5}$, etc., in Table II and the last column gives the number of basis vectors, which span the various irreducible representations. $\epsilon=\exp \left(\frac{1}{3} i 2 \pi\right)$, $\epsilon *=\exp \left(-\frac{1}{3} i 2 \pi\right)$.

\begin{tabular}{|c|c|c|c|c|c|c|c|c|}
\hline \multicolumn{9}{|c|}{$\begin{array}{l}K: \bar{k}=\left[\frac{1}{3}, \frac{1}{3}, 0\right] \\
\Gamma: \bar{k}=\left[\begin{array}{ll}0, & 0,0\end{array}\right]\end{array}$} \\
\hline$\Gamma, K$ & 1 & 3 & 5 & & 7 & 9 & 11 & No. of patterns spanned \\
\hline$\Gamma_{1}, K_{1}$ & 1 & 1 & 1 & & 1 & 1 & 1 & 1 \\
\hline$\Gamma_{2}, K_{2}$ & 1 & 1 & 1 & & -1 & -1 & -1 & 2 \\
\hline$\Gamma_{3}, K_{3}$ & $\begin{array}{ll}1 & 0 \\
0 & 1\end{array}$ & $\begin{array}{ll}\epsilon & 0 \\
0 & \epsilon *\end{array}$ & $\begin{array}{l}\epsilon^{*} \\
0\end{array}$ & $\begin{array}{l}0 \\
\epsilon\end{array}$ & $\begin{array}{ll}0 & 1 \\
1 & 0\end{array}$ & $\begin{array}{cc}0 & \epsilon \\
\epsilon^{*} & 0\end{array}$ & $\begin{array}{ll}0 & \epsilon * \\
\epsilon & 0\end{array}$ & $3 \times 2=6$ \\
\hline
\end{tabular}

$\Delta: \bar{k}=[0,0, \xi] ; 0<\xi<0.5$

$\left.P: \bar{k}=1 \frac{1}{3}, \frac{1}{3}, \xi\right] ; 0<\xi<0.5$

\begin{tabular}{llllc}
$\Delta, P$ & 1 & 3 & 5 & No. of patterns spanned \\
\hline$\Delta_{1}, P_{1}$ & 1 & 1 & 1 & 3 \\
$\Delta_{2}, P_{2}$ & 1 & $\epsilon$ & $\epsilon^{*}$ & 3 \\
$\Delta_{3}, P_{3}$ & 1 & $\epsilon^{*}$ & $\epsilon$ & 3 \\
\hline
\end{tabular}

$A: \bar{k}=[0,0,0.5]$

$H: \bar{k}=\left[\frac{1}{3}, \frac{1}{3}, 0.5\right]$

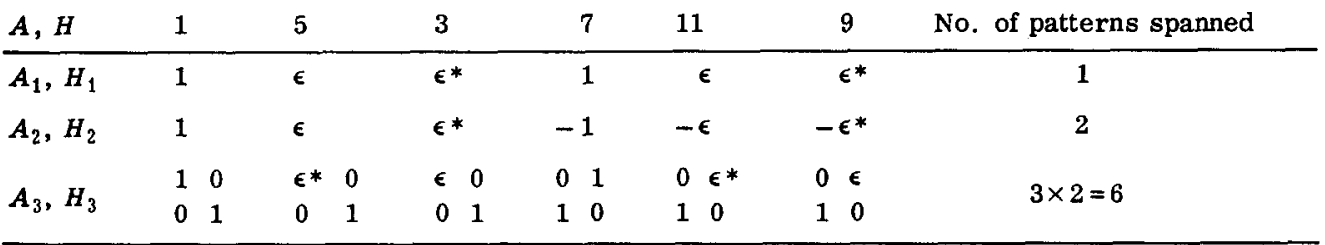

$T: \bar{k}=[\xi, \xi, 0] ; 0<\xi<\frac{1}{3}$

\begin{tabular}{cccc}
$T$ & 1 & 11 & No. of patterns spanned \\
\hline$T_{1}$ & 1 & 1 & 4 \\
$T_{2}$ & 1 & -1 & 5 \\
\hline
\end{tabular}

$S: \bar{k}=[\xi, \xi, 0.5] ; 0<\xi<\frac{1}{3}$

\begin{tabular}{llrc}
$S$ & $h_{1}$ & $h_{11}$ & No. of patterns spanned \\
\hline$S_{1}$ & 1 & $\epsilon$ & 4 \\
$S_{2}$ & 1 & $-\epsilon$ & 5 \\
\hline
\end{tabular}

$T^{\prime}: \bar{k}=[\xi,(1-2 \xi), 0]$

$M: \vec{k}=[0.5,0,0]$

\begin{tabular}{cccc}
\hline$M, T^{\prime}$ & $h_{1}$ & $h_{7}$ & No. of patterns spanned \\
$M_{1}, T_{1}^{\prime}$ & 1 & 1 & 4 \\
$M_{2}, T_{2}^{\prime}$ & 1 & -1 & 5 \\
\hline
\end{tabular}

$A_{1}$ frequency. From the $A_{2}$ block is seen that only coordinates $2,5,6$, and 8 make contributions. At $K$, the lower $K_{2}$ frequency is determined by coordinates 12 and 13 , whereas the high $K_{2}$ frequency is determined by coordinates $6,7,8$, and 9 . On this background it is fairly easy to set up a reasonable trial set of force constants, and let the normal mode programs optimize the set. The results are given in Tables III and IV.

VII. ELASTICITY MODULI AND SOUND VELOCITIES

The stress-strain relation for elastic solids is given according to

$$
\sigma_{i k}=\sum_{l, m} \lambda_{i k l m} \mu_{l m},
$$

where $\sigma_{1 k}$ is the stress tensor, $\mu_{1 m}$ is the strain tensor, and $\lambda_{i k l m}$ is the stiffness or elasticity tensor. The indices $i, k, l, m$ indicate the various Cartesian components and in the ordinary Voigt notation

$$
\begin{array}{ccccccc}
i k=11 & 22 & 33 & 23 & 13 & 12 & j 1 \sim x, 2 \sim y, 3 \sim z \\
\gamma=1 & 2 & 3 & 4 & 5 & 6 &
\end{array}
$$

Eq. (7. 1) may be written in matrix form $\sigma=c \mu$, 
TABLE VI. Symmetry coordinate matrix for the $\Gamma \rightarrow \Delta \rightarrow A$ direction. The first three elements in the $j$ th column show the displacements of atom 1 for the $j$ th symmetry coordinate, etc. The symmetries are shown by crosses. $f_{1}=$ $=\exp \left(\frac{1}{3} i \mathrm{k} \cdot 2 a_{3}\right), f_{2}=\exp \left(\frac{1}{3} i \mathbf{k} \cdot a_{3}\right), \epsilon=\exp \left(\frac{1}{3} i 2 \pi\right)$. A superscript ${ }^{*}$ indicates the complex conjugate.

\begin{tabular}{|c|c|c|c|c|c|c|c|c|c|}
\hline & $\Gamma-\Delta \rightarrow$ & & & & & & & & \\
\hline & $\frac{\sqrt{3}}{3}$ & 0 & 0 & $\frac{\sqrt{3}}{3}$ & 0 & 0 & $\frac{\sqrt{3}}{3}$ & 0 & 0 \\
\hline & 0 & $\frac{\sqrt{3}}{3}$ & 0 & 0 & $\frac{\sqrt{3}}{3}$ & 0 & 0 & $\frac{\sqrt{3}}{3}$ & 0 \\
\hline & 0 & 0 & $\frac{\sqrt{3}}{3}$ & 0 & 0 & $\frac{\sqrt{3}}{3}$ & 0 & 0 & $\frac{\sqrt{3}}{3}$ \\
\hline & $-\frac{\sqrt{3}}{6} f_{2}$ & $-\frac{1}{2} f_{2}$ & 0 & $-\frac{\sqrt{3}}{6} f_{2} \epsilon^{*}$ & $-\frac{1}{2} f_{2} \epsilon^{*}$ & 0 & $-\frac{\sqrt{3}}{6} f_{2} \epsilon$ & $-\frac{1}{2} f_{2} \epsilon$ & 0 \\
\hline & $\frac{1}{2} f_{2}$ & $-\frac{\sqrt{3}}{6} f_{2}$ & 0 & $\frac{1}{2} f_{2} \epsilon^{*}$ & $-\frac{\sqrt{3}}{6} f_{2} \in *$ & 0 & $\frac{1}{2} f_{2} \epsilon$ & $-\frac{\sqrt{3}}{2} f_{2} \epsilon$ & 0 \\
\hline & 0 & 0 & $\frac{\sqrt{3}}{3} f_{2}$ & 0 & 0 & $\frac{\sqrt{3}}{3} f_{2} \epsilon^{*}$ & 0 & 0 & $\frac{\sqrt{3}}{3} f_{2} \epsilon$ \\
\hline & $-\frac{\sqrt{3}}{6} f_{1}$ & $\frac{1}{2} f_{1}$ & 0 & $-\frac{\sqrt{3}}{6} f_{1} \epsilon$ & $\frac{1}{2} f_{1} \epsilon$ & 0 & $-\frac{\sqrt{3}}{6} f_{1} \epsilon^{*}$ & $\frac{1}{2} f_{1} \in *$ & 0 \\
\hline & $-\frac{1}{2} f_{1}$ & $-\frac{\sqrt{3}}{6} f_{1}$ & 0 & $-\frac{1}{2} f_{1} \epsilon$ & $-\frac{\sqrt{3}}{6} f_{1} \epsilon$ & 0 & $-\frac{1}{2} f_{1} \epsilon *$ & $-\frac{\sqrt{3}}{6} f_{1} \epsilon^{*}$ & 0 \\
\hline & 0 & 0 & $\frac{\sqrt{3}}{2} f_{1}$ & 0 & 0 & $\frac{\sqrt{3}}{3} f_{1} \epsilon$ & 0 & 0 & $\frac{\sqrt{3}}{3} f_{1} \in *$ \\
\hline$\Delta_{1}$ & $\mathrm{x}$ & $\mathbf{x}$ & $\mathbf{x}$ & & & & & & \\
\hline$\Delta_{2}$ & & & & $x$ & $x$ & $x$ & & & \\
\hline$\Delta_{3}$ & & & & & & & $\mathbf{x}$ & $\mathrm{x}$ & $x$ \\
\hline$\Gamma_{1}$ & $x$ & & & & & & & & \\
\hline$\Gamma_{2}$ & & $\mathrm{x}$ & $x$ & & & & & & \\
\hline$\Gamma_{3}$ & & & & $x$ & $x$ & $\mathbf{x}$ & $x$ & $x$ & $\mathrm{x}$ \\
\hline$A_{1}$ & & & & $\mathrm{x}$ & & & & & \\
\hline$A_{2}$ & & & & & $\mathbf{x}$ & $x$ & & & \\
\hline$A_{3}$ & $x$ & $x$ & $x$ & & & & $x$ & $x$ & $\mathrm{x}$ \\
\hline
\end{tabular}

$$
\left[\begin{array}{l}
\sigma_{1} \\
\sigma_{2} \\
\sigma_{3} \\
\sigma_{4} \\
\sigma_{5} \\
\sigma_{6}
\end{array}\right]=\left[\begin{array}{llll}
C_{11} & C_{12} & \cdots & C_{16} \\
C_{12} & C_{22} & \cdots & C_{26} \\
\cdot & & & \\
\cdot & & & \\
\cdot & & & \\
\cdot & & & \\
C_{16} & \ldots \ldots & \ldots & C_{66}
\end{array}\right]\left[\begin{array}{l}
\mu_{1} \\
\mu_{2} \\
\mu_{3} \\
\mu_{4} \\
\mu_{5} \\
\mu_{6}
\end{array}\right]
$$

The symmetry properties of selenium reduce the number of independent matrix elements in $\mathbf{C}$ from 21 to 6 according to

$$
\mathbf{c}=\left[\begin{array}{cccccc}
C_{11} & C_{12} & C_{13} & C_{14} & 0 & 0 \\
C_{12} & C_{11} & C_{13} & -C_{14} & 0 & 0 \\
C_{13} & C_{13} & C_{33} & 0 & 0 & 0 \\
C_{14} & -C_{14} & 0 & C_{44} & 0 & 0 \\
0 & 0 & 0 & 0 & C_{44} & C_{14} \\
0 & 0 & 0 & 0 & C_{14} & \frac{1}{2}\left(C_{11}-C_{12}\right)
\end{array}\right] .
$$

The equation of motion for the crystal in the $k \rightarrow 0$ limit leads to $3 \times 3$ secular equations from which the squared frequencies $\nu^{2}$ may be determined. The coefficient matrix for the strains $\mu_{r}$ may conveniently be written, following Nakayama and Odajima, ${ }^{6}$ 
TABLE VII. Symmetry coordinate matrix for the $\Gamma \rightarrow T \rightarrow K$ direction.

\begin{tabular}{|c|c|c|c|c|c|c|c|c|c|}
\hline & $\Gamma \rightarrow T$ & & & & & & & & \\
\hline & $\frac{\sqrt{3}}{3}$ & 0 & 0 & $\frac{\sqrt{6}}{6}$ & $\frac{\sqrt{2}}{2}$ & 0 & 0 & 0 & 0 \\
\hline & 0 & $\frac{\sqrt{2}}{2}$ & 0 & 0 & 0 & $\frac{\sqrt{3}}{3}$ & 0 & $\frac{\sqrt{6}}{6}$ & 0 \\
\hline & 0 & 0 & $\frac{\sqrt{2}}{2}$ & 0 & 0 & 0 & $\frac{\sqrt{3}}{3}$ & 0 & $\frac{\sqrt{6}}{6}$ \\
\hline & $-\frac{\sqrt{3}}{6}$ & $\frac{\sqrt{6}}{4}$ & 0 & $-\frac{\sqrt{6}}{12}$ & $\frac{\sqrt{2}}{4}$ & $-\frac{1}{2}$ & 0 & $-\frac{\sqrt{2}}{4}$ & 0 \\
\hline & $\frac{1}{2}$ & $\frac{\sqrt{2}}{4}$ & 0 & $\frac{\sqrt{2}}{4}$ & $-\frac{\sqrt{6}}{4}$ & $-\frac{\sqrt{3}}{6}$ & 0 & $-\frac{\sqrt{6}}{12}$ & 0 \\
\hline & 0 & 0 & $-\frac{\sqrt{2}}{2}$ & 0 & 0 & 0 & $\frac{\sqrt{3}}{3}$ & 0 & $\frac{\sqrt{6}}{6}$ \\
\hline & $-\frac{\sqrt{3}}{6}$ & 0 & 0 & $\frac{\sqrt{6}}{6}$ & 0 & $\frac{1}{2}$ & 0 & $-\frac{\sqrt{2}}{2}$ & 0 \\
\hline & $-\frac{1}{2}$ & 0 & 0 & $\frac{\sqrt{2}}{2}$ & 0 & $-\frac{\sqrt{3}}{6}$ & 0 & $\frac{\sqrt{6}}{6}$ & 0 \\
\hline & 0 & 0 & 0 & 0 & 0 & 0 & $\frac{\sqrt{3}}{3}$ & 0 & $-\frac{\sqrt{6}}{3}$ \\
\hline$T_{1}$ & $\mathrm{x}$ & $\mathrm{x}$ & $\mathbf{x}$ & $x$ & & & & & \\
\hline$T_{2}$ & & & & & $\mathbf{x}$ & $\mathbf{x}$ & $x$ & $\mathrm{x}$ & $\mathrm{x}$ \\
\hline$\Gamma_{1}$ & $x$ & & & & & & & & \\
\hline$\Gamma_{2}$ & & & & & & $\mathrm{x}$ & $x$ & & \\
\hline$\Gamma_{3}$ & & $x$ & $\mathbf{x}$ & $\mathbf{x}$ & $x$ & & & $\mathrm{x}$ & $x$ \\
\hline$K_{1}$ & $\mathrm{x}$ & & & & & & & & \\
\hline$K_{2}$ & & & & & & $\mathbf{x}$ & $\mathrm{x}$ & & \\
\hline$K_{3}$ & & $\mathbf{x}$ & $\mathrm{x}$ & $\mathrm{x}$ & $x$ & & & $\mathrm{x}$ & $\mathbf{x}$ \\
\hline
\end{tabular}

$\begin{gathered}(1,1) \\ (2,2) \\ (3,3) \\ (2,3) \\ (1,3) \\ (1,2)\end{gathered}\left[\begin{array}{cccccc}C_{11} & \frac{1}{2}\left(C_{11}-C_{12}\right) & C_{44} & C_{14} & 0 & 0 \\ \frac{1}{2}\left(C_{11}-C_{12}\right) & C_{11} & C_{44} & -C_{14} & 0 & 0 \\ C_{44} & C_{44} & C_{33} & 0 & 0 & 0 \\ C_{14} & -C_{14} & 0 & \frac{1}{2}\left(C_{13}+C_{44}\right) & 0 & 0 \\ 0 & 0 & 0 & 0 & \frac{1}{2}\left(C_{13}+C_{44}\right) & C_{14} \\ 0 & 0 & 0 & 0 & C_{14} & \frac{1}{4}\left(C_{11}+C_{12}\right)\end{array}\right]\left[\begin{array}{c}\kappa_{x}^{2} \\ \kappa_{y}^{2} \\ \kappa_{z}^{2} \\ 2 \kappa_{y} \kappa_{z} \\ 2 \kappa_{x} \kappa_{z} \\ 2 \kappa_{x} \kappa_{y}\end{array}\right]$.

We obtain essentially the same results as Nakayama et al. aside from apparently two typographical errors in their work. The indices $(i, j)$ to the left of each row in the matrix indicates that the number obtained by multiplying the respective row in the matrix with the column vector to the right is the $(i, j)^{\prime}$ the matrix element of the secular determinant, which is obtained by subtracting $\rho 4 \pi^{2} \nu^{2}$ from the diagonal elements. $\rho$ is the macroscopic density of the solid. We may now proceed and calculate the frequencies in any direction $\kappa$ and from this determine the elastic moduli. As we shall see, the results depend critically on the results obtained from the directions along $b_{3}$ and $b_{1}+b_{2}$. For $k=\xi b_{3}=\left(2 \pi /\left|a_{3}\right|\right) k$ $=\kappa_{k} \mathrm{k}$ it is seen that the secular determinant is diagonal with two identical roots corresponding to two degenera te transverse modes and a single root corresponding to a longitudinal mode. Our calculations show that below $\xi$ $=0.02$ the two lower modes merge together in agreement with the statement above. From the degenerate roots we find

$$
(\nu / \xi)_{\zeta \rightarrow 0}=2.8 \times 10^{12} \mathrm{cps} .
$$

With $\left|a_{3}\right|=4.95 \AA$ and $\rho=4.8 \mathrm{~g} / \mathrm{cm}^{3}$, we finally find $C_{44}=0.92 \mathrm{dyn} / \mathrm{cm}^{2}$ as compared to the observed value

$1.82 \times 10^{11} \mathrm{dyn} / \mathrm{cm}^{2}$. From the single root we find

$$
(\nu / \xi)_{\xi-0}=9.4 \times 10^{12} \mathrm{cps},
$$

which gives $C_{33}=10.4 \times 10^{11} \mathrm{dyn} / \mathrm{cm}^{2}$ as compared to the 
TABLE VIII. Symmetry coordinate matrix for the $A \rightarrow S \rightarrow H$ direction.

\begin{tabular}{|c|c|c|c|c|c|c|c|c|c|}
\hline & $A \rightarrow S$ & & & & & & & & \\
\hline & $\frac{\sqrt{3}}{3}$ & 0 & 0 & $\frac{\sqrt{6}}{6}$ & $\frac{\sqrt{2}}{2}$ & 0 & 0 & 0 & 0 \\
\hline & 0 & $\frac{\sqrt{2}}{2}$ & 0 & 0 & 0 & $\frac{\sqrt{3}}{3}$ & 0 & $\frac{\sqrt{6}}{6}$ & 0 \\
\hline & 0 & 0 & $\frac{\sqrt{2}}{2}$ & 0 & 0 & 0 & $\frac{\sqrt{3}}{3}$ & 0 & $\frac{\sqrt{6}}{6}$ \\
\hline & $\frac{\sqrt{3}}{6}$ & $-\frac{\sqrt{6}}{4}$ & 0 & $\frac{\sqrt{6}}{12}$ & $-\frac{\sqrt{2}}{4}$ & $\frac{1}{2}$ & 0 & $\frac{\sqrt{2}}{4}$ & 0 \\
\hline & $-\frac{1}{2}$ & $-\frac{\sqrt{2}}{4}$ & 0 & $-\frac{\sqrt{2}}{4}$ & $\frac{\sqrt{6}}{4}$ & $\frac{\sqrt{3}}{6}$ & 0 & $\frac{\sqrt{6}}{12}$ & 0 \\
\hline & 0 & 0 & $\frac{\sqrt{2}}{2}$ & 0 & 0 & 0 & $-\frac{\sqrt{3}}{3}$ & 0 & $-\frac{\sqrt{6}}{3}$ \\
\hline & $-\frac{\sqrt{3}}{6}$ & 0 & 0 & $\frac{\sqrt{6}}{6}$ & 0 & $\frac{1}{2}$ & 0 & $-\frac{\sqrt{2}}{2}$ & 0 \\
\hline & $-\frac{1}{2}$ & 0 & 0 & $\frac{\sqrt{2}}{2}$ & 0 & $-\frac{\sqrt{3}}{6}$ & 0 & $\frac{\sqrt{6}}{6}$ & 0 \\
\hline & 0 & 0 & 0 & 0 & 0 & 0 & $\frac{\sqrt{3}}{3}$ & 0 & $-\frac{\sqrt{6}}{3}$ \\
\hline$S_{1}$ & $\mathrm{x}$ & $x$ & $\mathbf{x}$ & $x$ & & & & & \\
\hline$S_{2}$ & & & & & $\mathrm{x}$ & $x$ & $x$ & $\mathrm{x}$ & $x$ \\
\hline$A_{1}$ & $\mathrm{x}$ & & & & & & & & \\
\hline$A_{2}$ & & & & & & $\mathrm{x}$ & $x$ & & \\
\hline$A_{3}$ & & $x$ & $\mathrm{x}$ & $x$ & $x$ & & & $\mathrm{x}$ & $\mathbf{x}$ \\
\hline$H_{1}$ & $x$ & & & & & & & & \\
\hline $\mathrm{H}_{2}$ & & & & & & $\mathrm{x}$ & $x$ & & \\
\hline$H_{3}$ & & $x$ & $\mathbf{x}$ & $x$ & $x$ & & & $\mathrm{x}$ & $x$ \\
\hline
\end{tabular}

observed value $8.2 \times 10^{11} \mathrm{dyn} / \mathrm{cm}^{2} .^{8,24}$ The $C_{33}$ is in fair agreement with experiments, but $C_{44}$ is much too low. We have not found it possible to adjust force constants to improve this situation without seriously reducing the rather good agreement for the optical branches. It seems that the force model of Wendel et al. ${ }^{5}$ also would have this problem. The slopes estimated from their acoustical branches along $\kappa=\xi b_{3}$ are roughly

$(\nu / \xi)_{\xi \rightarrow 0} \simeq 2.7 \times 10^{12} \mathrm{cps}$ and $(\nu / \xi)_{\xi \rightarrow 0} \simeq 7.4 \times 10^{12} \mathrm{cps}$, which leads, respectively, to,

$C_{44} \simeq 0.86 \times 10^{11} \mathrm{dyn} / \mathrm{cm}^{2}$ and $C_{33} \simeq 6.5 \times 10^{11} \mathrm{dyn} / \mathrm{cm}^{2}$.

The poor value of $C_{44}$ makes it impossible to calculate good values for the other $C_{i j}$ by considering other directions. For example, when $\kappa=\xi\left(b_{1}+b_{2}\right)$ a $3 \times 3$ secular determinant is obtained. It can be block diagonalized into a $1 \times 1$ and a $2 \times 2$ block by a similarity transfor mation. The branch with the highest root yields

$$
(\nu / \xi)_{\xi \rightarrow 0}=9.12 \times 10^{12} \mathrm{cps},
$$

which gives $C_{11}=1.91 \times 10^{11} \mathrm{dyn} / \mathrm{cm}^{2}$ as compared to the observed value $1.91 \times 10^{11} \mathrm{dyn} / \mathrm{cm}^{2}$. The $2 \times 2$ block in the secular equation yields $\frac{1}{2}\left(C_{11}-C_{12}\right)$ through the equation

$$
\frac{1}{2}\left(C_{11}-C_{12}\right)=\frac{\zeta\left|a_{1}\right|^{2}}{4}\left[\left(\frac{\nu^{+}}{\xi}\right)_{\xi-0}^{2}+\left(\frac{\nu^{-}}{\xi}\right)_{\xi-0}^{2}\right]-C_{44},
$$

where $\nu^{+}$and $\nu^{-}$are for the middle and lowest branches, respectively, along $(\xi, \xi, 0)$. Our calculations give

$$
\begin{aligned}
& \left(\nu^{+} / \xi\right)_{\xi \rightarrow 0}=6.20 \times 10^{12} \mathrm{cps}, \\
& \left(\nu^{-} / \xi\right)_{\xi-0}=3.94 \times 10^{12} \mathrm{cps},
\end{aligned}
$$

which result in $\frac{1}{2}\left(C_{11}-C_{12}\right)=0.25 \times 10^{11} \mathrm{dyn} / \mathrm{cm}^{2}$ as compared to the observed value $0.82 \times 10^{11} \mathrm{dyn} / \mathrm{cm}^{2}$. The $C_{14}$ constant may be obtained from

$$
C_{14}^{2}=\frac{1}{2}\left(C_{11}-C_{12}\right) C_{44}-\left(\frac{\rho\left|\mathbf{a}_{1}\right|^{2}}{4}\right)\left(\frac{\nu^{+}}{\xi} \frac{\nu^{-}}{\xi}\right)_{\xi \rightarrow 0}^{2},
$$

which yields an imaginary value for $C_{14}$. The problem arises because the value determined for $\frac{1}{2}\left(C_{11}-C_{12}\right)$ and $C_{44}$ are too small compared with experiments.

Finally, the sound velocities for various directions are given in Table $\mathrm{X}$.

\section{RESULTS AND DISCUSSION}

The calculated dispersion relations are shown in Figs. 4 and 5 where the experimental results also are shown. The symmtries are those found in the computa- 
TABLE IX. Symmetry coordinate matrix for the $K \rightarrow T^{\prime} \rightarrow M$ direction.

\begin{tabular}{|c|c|c|c|c|c|c|c|c|c|}
\hline & \multicolumn{9}{|c|}{$K \rightarrow T^{\prime} \rightarrow M$} \\
\hline & $\frac{\sqrt{3}}{3}$ & 0 & 0 & $\frac{\sqrt{6}}{6}$ & $\frac{\sqrt{2}}{2}$ & 0 & 0 & 0 & 0 \\
\hline & 0 & $\frac{\sqrt{2}}{2}$ & 0 & 0 & 0 & $\frac{\sqrt{3}}{3}$ & 0 & $\frac{\sqrt{6}}{6}$ & 0 \\
\hline & 0 & 0 & $\frac{\sqrt{2}}{2}$ & 0 & 0 & 0 & $\frac{\sqrt{3}}{3}$ & 0 & $\frac{\sqrt{6}}{6}$ \\
\hline & $-\frac{\sqrt{3}}{6}$ & 0 & 0 & $\frac{\sqrt{6}}{6}$ & 0 & $-\frac{1}{2}$ & 0 & $\frac{\sqrt{2}}{2}$ & 0 \\
\hline & $\frac{1}{2}$ & 0 & 0 & $-\frac{\sqrt{2}}{2}$ & 0 & $-\frac{\sqrt{3}}{6}$ & 0 & $\frac{\sqrt{6}}{6}$ & 0 \\
\hline & 0 & 0 & 0 & 0 & 0 & 0 & $\frac{\sqrt{3}}{3}$ & 0 & $-\frac{\sqrt{3}}{6}$ \\
\hline & $-\frac{\sqrt{3}}{6}$ & $-\frac{\sqrt{6}}{4}$ & 0 & $-\frac{\sqrt{6}}{12}$ & $\frac{\sqrt{2}}{2}$ & $\frac{1}{2}$ & 0 & $\frac{\sqrt{2}}{4}$ & 0 \\
\hline & $-\frac{1}{2}$ & $\frac{\sqrt{2}}{4}$ & 0 & $-\frac{\sqrt{2}}{4}$ & $\frac{\sqrt{6}}{4}$ & $-\frac{\sqrt{2}}{2}$ & 0 & $-\frac{\sqrt{6}}{12}$ & 0 \\
\hline & 0 & 0 & $-\frac{\sqrt{2}}{2}$ & 0 & 0 & 0 & $\frac{\sqrt{3}}{3}$ & 0 & $\frac{\sqrt{6}}{6}$ \\
\hline$T_{1}^{\prime}$ & $\mathbf{x}$ & $\mathbf{x}$ & $\mathbf{x}$ & $\mathbf{x}$ & & & & & \\
\hline$T_{2}^{\prime}$ & & & & & $\mathbf{x}$ & $\mathbf{x}$ & $\mathbf{x}$ & $\mathbf{x}$ & $\mathbf{x}$ \\
\hline$K_{1}$ & $\mathrm{x}$ & & & & & & & & \\
\hline$K_{2}$ & & & & & & $\mathbf{x}$ & $\mathrm{x}$ & & \\
\hline$K_{3}$ & & $\mathbf{x}$ & $\mathbf{x}$ & $\mathbf{x}$ & $\mathrm{x}$ & & & $x$ & $\mathbf{x}$ \\
\hline$M_{1}$ & $\mathrm{x}$ & $\mathbf{x}$ & $\mathbf{x}$ & $\mathbf{x}$ & & & & & \\
\hline$M_{2}$ & & & & & $\mathbf{x}$ & $\mathbf{x}$ & $\mathrm{x}$ & $\mathrm{x}$ & $\mathbf{x}$ \\
\hline
\end{tabular}

tions, and they agree with those of Teuchert et al. ${ }^{2}$ As the modes are arranged in three bands, let us comment them briefly separately.

\section{A. Upper optical band}

In general our results are seen to be in agreement with experimental results. Other models have failed in this region and just give flat bands. Wendel et al. ${ }^{5}$ tried to improve their combined valence potential-Keating potential model by introducing long range Coulombic forces, but obtained only relatively little improvement of their results. Along $\Delta$ and $P$ the dispersion is solely deter mined by intrachain forces. As mentioned in Sec. II only the introduction of the symmetrized dihedral tor sion coordinates made it possible to obtain a satisfactory dispersion of the upper modes. Physically, this probably means that due to the lone pairs of electrons along the chain, one has to use more complex coordinates than the simple valence coordinates, which work well for covalent bonded atoms. We have to include interactions between the rather distant atoms, here between five atoms along the chain. This is in fact what both Martin et al. ${ }^{8}$ and Wendel ${ }^{5}$ suggest for a possible improvement of their results.

Besides the intrachain coordinates themselves, the coupling terms between 1 and 3 and between 1 and 4 proved to be important for these modes, while the other coupling terms had no influence there. An analysis showed that the dispersion of the upper optic modes in directions at right angles to the screw axis primarily is due to coordinates 10 and 11.

\section{B. Lower optical modes}

This band is also fairly well described, although deviations from experimental results are noticed. The $\Gamma_{2} \rightarrow T_{2} \rightarrow K_{2}$ branch is determined by coordinates 6,7 ,

TABLE X. Sound velocities.

\begin{tabular}{lccc}
\hline$\kappa$ & $(\nu / \xi)_{\xi-0} * 10^{-12} \mathrm{cps} \AA$ & $v_{3} \times 10^{-5} \mathrm{~cm} / \mathrm{s}$ & $v_{s} \times 10^{-5}(\mathrm{obs})^{\mathrm{a}, \mathrm{b}}$ \\
\hline $00 \xi$ & 9.38 & 4.65 & 4.14 \\
& 2.80 & 1.39 & 1.95 \\
$\xi \xi 0$ & 9.12 & 1.99 & 1.98 \\
& 6.20 & 1.36 & 1.94 \\
& 3.94 & 0.86 & 0.745 \\
\hline
\end{tabular}

aSee Table I, Ref. 2.

beuchert et al. (Ref. 2) deduce the existence of three slopes along $(0,0, \xi)$ from their experimental data. The theory shows that two branches should merge at small $\xi$ along $b_{3}$. Evidently, their data are not precise enough to establish this behavior. 
and 8. The branch emerging from $\Gamma_{2}$ in the $\Delta$ direction is determined in a complicated way by the different force constants as is the other branches in this band. The couplings between coordinates 3 and 4 and between 2 and 5 are essential for the branches in this band.

\section{Acoustical modes}

We made an interesting observation concerning the acoustical modes in the $\Delta$ direction. It is easy to see from the symmetry coordinates that the $\Delta_{2}$ and $\Delta_{3}$ branches correspond to transverse acoustic modes. It became apparent that these modes could not be accounted for by the intrachain coordinates especially at small wave vectors. The sound velocities were too small. Decisive for these branches are the excitation of coordinate 8 , and this indicates that the transverse acoustic phonons along $\Delta$ to a large extent are due to interchain interactions rather than to intrachain interactions.

\section{CONCLUSION}

The objective of this work has been twofold. First we have applied a method where the symmetry of the problem is introduced into the definition of the force field as symmetrized coordinates. These have been projected from ordinary valence coordinates for the covalent bonded atoms, and from relative rotation and translation coordinates developed to describe interactions between nonbonded groups of atoms. Secondly, we have shown that these types of coordinates are well suited for the dynamics of trigonal selenium, where we treat the interactions between atoms in different chains as between atoms in different molecules in molecular crystals. In particular we were able to obtain the dis persion of the upper optic moles very easily, a feature it would have been difficult to obtain with an ordinary set of valence coordinates. Although the symmetry arguments for eliminating coupling terms are indisputable if the coordinates are projected from the full space group of the crystal, one should be careful not to end up with unphysical coordinates if this is done, In the case of selenium, which is a nonsymmorphic crystal, such coordinates involved interactions between very distant atoms. Since the forces are short ranged, a reasonable compromise is to base the arguments on the local symmetry, as we have done. The complicated interchain interactions are very difficult to handle in terms of valence force models, since so many angle bends and bond stretches are involved. The relative rotation and translation coordinates are much easier to apprehend and physically more appealing. When combined with the local symmetry of the groups, the constraint equations are easy and simple to set up. They seem to apply well in this problem.

\section{ACKNOWLEDGMENTS}

We are indebted to Dr. R. M. Brugger, Director of the Research Reactor Facility, University of Missouri, Columbia for his encouragement and support. The Physics Department of Idaho State University provided facilities helpful to the completion of the work. One of the authors (F.Y.H.) is indebted to the Danish Research Council, and to the Research Grants Programme of the Scientific Affairs Division of NATO for financial support.

${ }^{1}$ W. C. Hamilton, B. Lassier, and M. I, Kay, J. Phys. Chem. Solids 35, 1089-1094 (1974).

${ }^{2}$ W. D. Teuchert, R. Geick, G. Landwehr, H. Wendel, and W. Weber, J. Phys, C 8, 3725 (1975).

${ }^{3}$ M. Hulin, Ann. Phys. (Paris) 8, 647 (1963).

${ }^{4}$ R. Geick, U. Schrøder, and J. Stuke, Phys. Status Solidi 24, 99 (1967).

${ }^{5}$ H. Wendel, W. Weber, and W. D. Teuchert, J. Phys. C 8, 3737 (1975).

${ }^{6}$ T. Nakayama and A. Odajima, J, Phys. Soc. Jpn. 33, 12 (1972).

${ }^{7}$ T. Nakayama and A. Odajima, J. Phys. Soc. Jpn. 34, 732 (1973).

${ }^{8}$ R. Martin, G. Lucovsky, and K. Helliwell, Phys. Rev. B 13, 1383 (1976).

${ }^{9}$ P. N. Keating, Phys. Rev. 145, 637-645 (1966).

${ }^{10}$ H. L. McMurry and F. Y. Hansen, J. Chem. Phys. 72, 5540 (1980); preceding paper.

${ }^{11} \mathrm{~F}$. Y. Hansen, T. S. Knudsen, and K. Carneiro, J. Chem. Phys. 62, 1556 (1975).

${ }^{12} \mathrm{G}$ melins Handbuch der Anorganischen Chemie, Selen, Teil A, Seite 187 (Springer Verlag, Berlin, 1924).

${ }^{13}$ D. W. Scott, J. P. McCullough, and F. H. Krause, J, Mol. Spectrosc. 13, 313 (1964).

${ }^{14}$ A. J. Bradley, Philos. Mag. 48, 477 (1924).

${ }^{15}$ P. J. Flory, Statistical Mechanics of Chain Molecules (Interscience, New York, 1969), pp. 157-158.

${ }^{16}$ D. R. McCann and L. Cartz, J. Chem. Phys. 56, 2552 (1972).

${ }^{17} \mathrm{~J}$. C. Jamieson and D. B. McWahn, J. Chem. Phys. 43, 1149 (1965)

${ }^{18}$ F. Y. Hansen, Comput. Phys. Commun. 14, 193-218 (1978); 14, 219-243 (1978); and 14, 245-254 (1978).

${ }^{19}$ F. Y. Hansen, Phys. Rev. B 18, 4015-4038 (1978).

${ }^{20} \mathrm{E}$. B. Wilson, Jr., J. C. Decius, and P. C. Cross, Molecular Vibrations (McG raw-Hill, New York, 1955).

${ }^{21}$ P. Herman, J. Phys. Chem. Solids 8, 405 (1959).

${ }^{22} \mathrm{O}$. V. Kovalev, Irreducible Representations of the Space $G$ roups (Gordon and Breach, New York, 1965).

${ }^{23} \mathrm{G}$. F. Koster, Solid State Phys. 5, $173(1957)$.

${ }^{24}$ J. Mort, J. Appl. Phys. 38, 3414 (1967).

${ }^{25}$ M. E. Swanson, N. T. Gilfích, and G. M. Ugrinic, Natl. Bur. Stand. Circ. No. 539, 54 (1955).

${ }^{26}$ R. W. G. Wyckoff, Crystal Structures (Interscience, New York, 1963), p. 36.

${ }^{27}$ P. Grosse, A. Svoboda, and J. Tousand, J. Phys. Chem. Solids 21, 445 (1975).

${ }^{28} \mathrm{P}$. Cherin and P. Unger, Acta Crystallogr. 21, 000 (1966); Inorg. Chem. 6, 1589 (1967). 